nephron

Practice
Nephron 2017;136:221-225

DOI: $10.1159 / 000468547$
Received: November 11, 2016

Accepted after revision: March 3, 2017

Published online: April 8, 2017

\title{
Interpretation of the Fractional Excretion of Sodium in the Absence of Acute Kidney Injury: A Cross-Sectional Study
}

\author{
Michiel F. Schreuder ${ }^{\mathrm{a}}$ Arend Bökenkamp ${ }^{\mathrm{b}}$ Joanna A.E. van Wijk ${ }^{\mathrm{b}}$

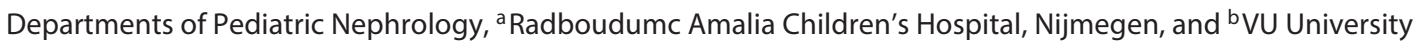 \\ Medical Center, Amsterdam, The Netherlands
}

\section{Keywords}

Fractional excretion of sodium - Glomerular filtration rate . Sodium excretion $\cdot$ Renal tubular function

\begin{abstract}
Background/Aims: The fractional excretion of sodium (FeNa) may be helpful in establishing the cause of acute renal failure. This study was performed to determine the influence of the glomerular filtration rate (GFR), sodium intake, and tubular function on FeNa in children without renal failure. Methods: In this single institute cross-sectional study, 24-h-urine collections from patients (4-18 years of age, GFR $>60 \mathrm{~mL} / \mathrm{min} / 1.73 \mathrm{~m}^{2}$ ) were used when considered reliable, and analyzed to determine sodium excretion, creatinine clearance and FeNa. The influence of tubular function was studied in 5 patients with generalized tubular dysfunction. Results: Based on data from 761 patients, a multiple regression formula was designed based on GFR and sodium excretion that predicted over $80 \%$ of the variation in FeNa $\left(R^{2}=\right.$ $0.824, p<0.001)$. Using this formula, the predicted FeNa was significantly lower than the measured FeNa in the children with tubular dysfunction. Conclusion: FeNa depends on GFR and sodium intake, and tubular function cannot be assessed
\end{abstract}

\section{KARGER}

E-Mail karger@karger.com www.karger.com/nef NonCommercial-NoDerivatives 4.0 International License (CC BYNC-ND) (http://www.karger.com/Services/OpenAccessLicense) Usage and distribution for commercial purposes as well as an
This article is licensed under the Creative Commons Attribution- without possessing adequate knowledge on all factors. Therefore, no normal range or cut-off value for FeNa to assess tubular function can be given, and doing so is a misapplication of this equation.

(c) 2017 The Author(s)

Published by S. Karger AG, Basel

\section{Introduction}

The fractional excretion of sodium $(\mathrm{FeNa})$ has been reported to be of value in determining the cause of acute kidney injury (AKI) [1, 2]. Nguyen et al. [3] described misapplications of commonly used kidney equations including $\mathrm{FeNa}$ and focused on the application and caveats of FeNa during AKI. In the absence of AKI, FeNa has been described to be influenced by daily sodium intake [4] and the glomerular filtration rate (GFR) [5].

It is unresolved whether FeNa can provide additional information, for instance, on tubular function, and which factors limit its use. Several papers have been published that describe $\mathrm{FeNa}$ as a marker of proximal tubular function without considering GFR or sodium intake $[6,7]$. It may, however, be argued that as long as a nor-

Dr. Michiel F. Schreuder

Department of Pediatric Nephrology

Radboud University Medical Center, Amalia Children's Hospital

PO Box 9101, NL-6500 HB Nijmegen (The Netherlands)

E-Mail michiel.schreuder@ radboudumc.nl 
mal serum level of sodium is maintained, renal sodium handling is functioning properly regardless of the level of FeNa.

In order to study the possible misapplication of $\mathrm{FeNa}$, we addressed the function and interpretation of $\mathrm{FeNa}$ in the absence of AKI and determined the influence of GFR, sodium intake, and tubular (dys)function on $\mathrm{FeNa}$, by analyzing 24-h-urine data from a cohort of children from our out-patient clinics.

\section{Subjects and Methods}

To study the influence of sodium excretion, as a marker of dietary sodium intake, and GFR (measured by $24 \mathrm{~h}$ urine creatinine clearance) on $\mathrm{FeNa}$, we performed a cross-sectional study and analyzed the results of 24-h-urine collections from the database of the Department of Pediatric Nephrology of the VU University Medical Center that were collected from our out-patient clinics between 2002 and 2008.

A single 24-h-urine collection per patient was used when considered reliable. We considered the urine collection reliable when both the nurse and the parents considered it to be reliable and without any missing portions. Also, strict criteria had to be met, based on the age of the patient (over 4 years), the collection period (between 20 and $28 \mathrm{~h}$ ), urinary creatinine excretion (between 88 and $265 \mu \mathrm{mol} / \mathrm{kg} / 24 \mathrm{~h} \mathrm{[8])} \mathrm{and} \mathrm{urine} \mathrm{volume} \mathrm{(between} 300$ and $\left.2,000 \mathrm{~mL} / \mathrm{m}^{2} / 24 \mathrm{~h}\right)$. Patients with documented diuretic use were excluded. Using this strict selection, a total of 761 out of 1,953 patients were selected, and the first reliable $24 \mathrm{~h}$ urine collections from these patients were used to calculate $24 \mathrm{~h}$ sodium excretion, cCreat and $\mathrm{FeNa}$, using formula 1:

$$
\mathrm{FeNa}(\%)=\frac{\mathrm{Na}_{\text {urine }}(\mathrm{mmol} / \mathrm{L}) \times \mathrm{Creat}_{\text {serum }}(\mu \mathrm{mol} / \mathrm{L})}{\mathrm{Na}_{\text {serum }}(\mathrm{mmol} / \mathrm{L}) \times \mathrm{Creat}_{\text {urine }}(\mathrm{mmol} / \mathrm{L}) \times 1,000} \times 100 \%
$$

This formula helps in the calculation of FeNa using random but simultaneous blood and urine measurements without the necessity of urine collections. However, the rationale behind formula 1 is based on:

$$
\begin{aligned}
\text { FeNa }(\%)= & \frac{\mathrm{Na}_{24 \text { h-excetion }}}{\mathrm{Na}_{24 \text { h-filtration }}} \times 100 \% \\
\text { FeNa }(\%)= & \frac{\mathrm{Na}_{24 \text { h-excretion }}(\mathrm{mmol} / \text { day })}{\mathrm{Na}_{\text {serum }}(\mathrm{mmol} / \mathrm{L}) \times \mathrm{GFR}\left(\mathrm{mL} / \mathrm{min} / 1.73 \mathrm{~m}^{2}\right) \times} \times 100 \% \\
& 1.44(\times 1,000 \mathrm{~min} / \text { day }) \times \mathrm{BSA}\left(\mathrm{m}^{2}\right) / 1.73
\end{aligned}
$$

Formula 2 shows that FeNa is expected to depend on both GFR and sodium excretion and thereby sodium intake. As a rule, GFR is corrected for body surface area (BSA) in pediatrics, but the amount of filtered sodium depends on the product of serum sodium and absolute or uncorrected GFR (hence, the factor [BSA/1.73] in formula $2 b$ ).

On the basis of predetermined cut-off levels of urinary sodium excretion, 4 groups were formed: group A with a sodium excretion of less than $50 \mathrm{mmol} /$ day $(<\sim 3 \mathrm{~g}$ salt/day), group B with a sodium
Table 1. Demographic data

Number

761

Male, \%

48.6

Age, years

$9.32(3.77)$

$1.12(0.34)$

Body surface area, $\mathrm{m}^{2}$

$99.1(22.7)$

Uncorrected creatinine clearance, $\mathrm{mL} / \mathrm{min}$

$65.3(27.9)$

Serum sodium, $\mathrm{mmol} / \mathrm{L}$

$139(4.3)$

$\mathrm{FeNa}, \%$

$0.76(0.45)$

$\mathrm{FeNa} \geq 1 \%, \%$

18.9

Sodium excretion, $\mathrm{mmol} /$ day

$96.8(70.0)$

Data are presented as number, percentage, or mean (SD). $\mathrm{FeNa}$, fractional excretion of sodium.

excretion of 50-100 mmol/day ( 3-6 g salt/day), group C with a sodium excretion of $100-150 \mathrm{mmol} /$ day ( $6-9 \mathrm{~g}$ salt/day), and group D with a sodium excretion of over $150 \mathrm{mmol} /$ day $(>\sim 9 \mathrm{~g}$ salt/day). Per group of sodium excretion, regression on the association between cCreat and FeNa was performed.

Creatinine was measured using the Jaffe method and sodium was measured using an ion selective electrode.

\section{Statistical Analysis}

Data is presented as mean (SD). Univariate linear regression and stepwise multiple regression were performed (probability of $F$ to enter $\leq 0.050$, probability of $F$ to remove $\geq 0.10$ ), and the regression coefficients are presented with $R^{2}$. Patients with missing data were excluded per analysis. SPSS version 11.0.1 (Windows, SPSS Inc., Chicago, IL, USA) was used for statistical analysis. A $p$ value of $<0.05$ was considered to be statistically significant.

\section{Results}

Patient characteristics and results from the urine collections are presented in Table 1. Figure 1 shows the association between uncorrected cCreat and $\mathrm{FeNa}$ for the 4 categories of sodium intake. As can be seen in Table 2, for all four categories of sodium intake, a highly significant association was present between cCreat and $\mathrm{FeNa}$ with cCreat explaining $48-85 \%$ of the variation in FeNa. However, for each category of sodium intake, the numerical relation with cCreat is different and analysis of cCreat was not significantly related to $\mathrm{FeNa}\left(r^{2}=0.029\right)$ in a univariate model not taking urinary sodium excretion into account.

Performing univariate linear regression with the factors age $\left(R^{2}=0.001, p=0.47\right)$, weight $\left(R^{2}=0.002, p=\right.$ $0.18)$, height $\left(R^{2}=0.002, p=0.21\right)$, BSA $\left(R^{2}=0.002, p=\right.$ $0.20)$, uncorrected cCreat $\left(R^{2}=0.017, p<0.001\right)$, and so- 
Table 2. Regression analysis between uncorrected creatinine clearance (cCreat) and fractional excretion of sodium (FeNa) per category of sodium intake

\begin{tabular}{lllll}
\hline $\begin{array}{l}\text { Urinary sodium excretion, } \\
\text { mmol/day }\end{array}$ & $N$ & Regression formula & $R^{2}$ & $p$ value \\
\hline $0-50$ & 137 & $\mathrm{FeNa}=1.00-\left(0.016^{*}\right.$ cCreat $)+\left(0.000073 *\right.$ cCreat $\left.^{2}\right)$ & 0.485 & $<0.001$ \\
$50-100$ & 338 & $\mathrm{FeNa}=1.55-(0.020 *$ cCreat $)+\left(0.000085 * \mathrm{cCreat}^{2}\right)$ & 0.486 & $<0.001$ \\
$100-150$ & 183 & $\mathrm{FeNa}=2.37-(0.029 *$ cCreat $)+\left(0.0001\right.$ cCreat $\left.^{2}\right)$ & 0.855 & $<0.001$ \\
$>150$ & 102 & $\mathrm{FeNa}=4.46-(0.056 *$ cCreat $)+\left(0.00020 * \mathrm{cCreat}^{2}\right)$ & 0.478 & $<0.001$ \\
All & 760 & $\mathrm{FeNa}=0.98-\left(0.0044^{*}\right.$ cCreat $)+\left(0.000012 *\right.$ cCreat $\left.^{2}\right)$ & 0.029 & $\mathrm{~ns}$ \\
\hline
\end{tabular}

ns, not significant.

Table 3. Renal functional data of children with generalized tubular dysfunction

\begin{tabular}{lllllll}
\hline Urinary sodium excretion & Patient A* & Patient B & Patient C & Patient D & Patient E & Mean (SD) \\
\hline Gender & Female & Male & Male & Male & Male & \\
Age, years & 0.45 & 16.3 & 13.1 & 7.3 & 8.2 & $9.1(6.1)$ \\
Diagnosis & Mito & TIN & TIN & Mito & NPH & \\
Creatinine clearance, $\mathrm{mL} / \mathrm{min} / 1.73 \mathrm{~m}^{2}$ & 30.4 & 23.1 & 9.7 & 33.9 & 60.5 & $31.5(18.7)$ \\
Uncorrected creatinine clearance, $\mathrm{mL} / \mathrm{min}$ & 5.1 & 23.0 & 8.2 & 15.0 & 23.4 & $14.9(8.4)$ \\
FeNa, \% & 2.5 & 1.7 & 9.4 & 7.2 & 6.2 & $5.4(3.2)$ \\
Estimated FeNa, \% & 0.9 & 1.0 & 1.8 & 2.0 & 2.5 & $1.6(0.7)$ \\
Sodium excretion, mmol/24 h & 24 & 75 & 161 & 209 & 290 & $152(106)$ \\
\hline
\end{tabular}

Mito, mitochondriopathy; TIN, tubulo-interstitial nephritis; NPH, nephronophthisis; FeNa, fractional excretion of sodium. * 24-h urine collection performed by bladder catheterization.

Fig. 1. Association between the fractional excretion of sodium (FeNa) and uncorrected creatinine clearance per category of sodium intake.

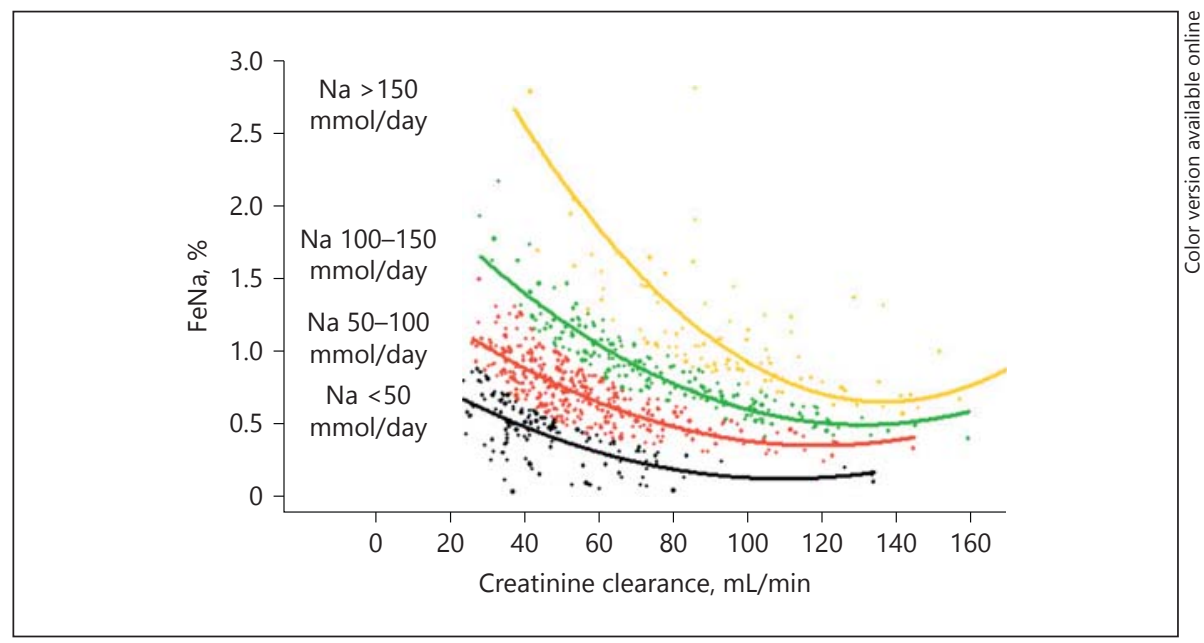

dium excretion $\left(R^{2}=0.539, p<0.001\right)$, only the latter 2 were included in the stepwise multiple regression model that explained more than $80 \%$ of the variation in $\mathrm{FeNa}$ $\left(r^{2}=0.824, p<0.001\right)$ :

$\begin{aligned} \text { FeNa }(\%)= & 0.77+(0.0066 \cdot \mathrm{Na} \text { excretion }[\mathrm{mmol} / \mathrm{day}]) \\ & -\left(0.0098 \cdot \mathrm{cCreat}_{\text {uncorrected }}[\mathrm{mL} / \mathrm{min}]\right)\end{aligned}$

Interpretation of $\mathrm{FeNa}$
To evaluate the influence of tubular function on $\mathrm{FeNa}$, 5 patients with generalized tubular dysfunction were identified in the study cohort (Table 3). Measured FeNa was significantly higher than the estimated $\mathrm{FeNa}$ based on formula 3 (mean difference 3.77 [95\% CI 0.28-7.26], $p=$ 0.04 ) illustrating that FeNa does reflect tubular (dys)function, but only after correction for GFR and sodium intake.

Nephron 2017;136:221-225 


\section{Discussion}

Our data show that FeNa depends on GFR and sodium intake. The use of FeNa to evaluate tubular function can be made only after correction is carried out for these 2 factors, and therefore, no cut-off level to distinguish between a normal and abnormal tubular sodium handling can be provided. The cut-off level of $1 \%$ that is used in the literature $[6,7]$ is therefore not suitable unless pre-renal AKI is diagnosed $[1,2]$.

As the level of FeNa depends on several important factors (glomerular filtration, tubular function and sodium intake), it cannot be used to interpret one of these factors without considering the others. FeNa is useful for the classification of AKI, as pre-renal failure volume depletion causes a maximal stimulus for salt retention and hence a low FeNa. In other clinical situations, however, defining any single cut-off value for FeNa is unhelpful for the diagnosis of tubular dysfunction. FeNa could be used to this end if the two other determinants (i.e., GFR and total sodium intake) are known and taken into account as demonstrated by the analysis of 5 patients with documented tubular dysfunction. By comparing expected $\mathrm{FeNa}$ on the basis of urinary sodium excretion and cCreat using formula 3 , a difference between the measured and predicted $\mathrm{FeNa}$ of more than $1 \%$ may be indicative of tubular dysfunction.

For practical reasons, it would be best to have a formula to predict FeNa solely on (an estimate of) GFR, as it would implicate that no urine collection is necessary. Unfortunately, the overall regression formula in Table 2 shows that such a formula would not be accurate, thereby restricting its value on forehand. Even when the analysis is limited to individuals with a sodium intake between 50 and $150 \mathrm{mmol} / \mathrm{day}$, as sodium intake in children is expected to be in this range [9], still only $33 \%$ of the variation in FeNa would be predicted (data not shown), compared with $82 \%$ when both GFR and sodium excretion are used (formula 3). This shows that for a proper interpretation of FeNa, knowledge of both GFR and sodium excretion is indispensable.

Our study has several limitations. First, $24 \mathrm{~h}$ urine collections were used to calculate the sodium and creatinine excretion, which is known to be unreliable in many instances. In order to try and optimise the reliability, we used strict inclusion criteria based on history and creatinine excretion. As collection of $24 \mathrm{~h}$ urine is done routinely at our institution, families are very familiar with this technique. Also, although imprecise urine collection may have introduced higher variability in our analysis, it should have no systematic effect on the relation between
GFR and urine sodium excretion in our analysis, both of which depend on urine collection.

We have chosen to present both sodium excretion and GFR without correction for weight or BSA, which is the usual approach in adults. As both anthropometric parameters are physiologically linked, correction for weight or BSA would have yielded comparable results.

Another limitation is that selection of patients cannot be excluded, as the patients in our study are derived from a hospital-based series. Even though we have tried to exclude patients using diuretics, database data on drug use may very well have been incomplete, allowing for some patients using diuretics to be included. In our experience, however, most patients will have been referred to the outpatient clinic of our department before such treatment has been initiated, thereby minimizing the possible influence of drug use on our results. The selection steps that resulted in the 761 patients included in the current analysis did change the characteristics of the cohort. With the exclusion of patients younger than 4 years of age, the mean age of the included group (9.32 [SD 3.77] years) was higher than the entire cohort (6.22 [SD 5.37] years). More boys were in the entire cohort (56.7\%) than in the cohort that was used for the current analysis (48.6\%; Table 1). Both changes were to be expected due to the exclusion of patients below the age of 4 years, in which an overrepresentation of boys is anticipated.

Only a few patients diagnosed with generalized tubular dysfunction fulfilled the criteria for inclusion based on missing data. These low numbers do not provide enough statistical power to establish a precise threshold for the difference between measured and calculated $\mathrm{FeNa}$ that is indicative of tubular dysfunction. Furthermore, the GFR in these 5 patients was lower than the GFR in the cohort from which the estimation formula was derived.

In conclusion, besides tubular function, $\mathrm{FeNa}$ depends on GFR and sodium intake. Therefore, tubular function cannot be assessed without the knowledge of the other 2 factors. Still, FeNa is useful for the classification of acute renal failure.

\section{Statement of Ethics}

As this was a retrospective analysis of anonymous data, no ethical approval was required.

\section{Disclosure Statement}

The authors declare no conflicts of interest.
Schreuder/Bökenkamp/van Wijk 


\section{References}

1 Espinel CH: The FENa test. Use in the differential diagnosis of acute renal failure. JAMA 1976;236:579-581.

2 Bhargava S, Jain A, Gupta V: Fractional excretion of sodium - a simple test for the differential diagnosis of acute renal failure. Clin Nephrol 2002;58:79-80.

3 Nguyen MT, Maynard SE, Kimmel PL: Misapplications of commonly used kidney equations: renal physiology in practice. Clin J Am Soc Nephrol 2009;4:528-534.
4 Heer M, Baisch F, Kropp J, Gerzer R, Drummer C: High dietary sodium chloride consumption may not induce body fluid retention in humans. Am J Physiol Renal Physiol 2000;278:F585-F595.

5 Kamel KS, Davids MR, Lin SH, Halperin ML: Interpretation of electrolyte and acid-base parameters in blood and urine; in Brenner BM (ed): Brenner and Rector's The Kidney. Philadelphia, Saunders Elsevier, 2008, pp 757-782.

6 Futrakul P, Kullavanijaya P, Watana D, Sensidivatana R, Kwakpetoon S, Unchumchoke P, Teranaparin C, Kheokham K: Tubular functions in glomerulonephropathies in childhood. Int J Pediatr Nephrol 1981;2:17-21.
7 Dagan A, Eisenstein B, Bar-Nathan N, Cleper R, Krause I, Smolkin V, Davidovits M: Tubular and glomerular function in children after renal transplantation. Pediatr Transplant 2005;9:440-444.

8 Hellerstein S, Simon SD, Berenbom M, Erwin $P$, Nickell E: Creatinine excretion rates for renal clearance studies. Pediatr Nephrol 2001; 16:637-643.

9 Schreuder MF, Bökenkamp A, van Wijk JA: Salt intake in children: increasing concerns? Hypertension 2007;49:e10; author reply e11. 